\title{
Synchronization and Control of Linearly Coupled Singular Systems
}

\author{
Fang Qingxiang, ${ }^{1,2}$ Peng Jigen, ${ }^{1}$ and Cao Feilong ${ }^{2}$ \\ ${ }^{1}$ School of Mathematics and Statistics, Xian Jiaotong University, Xian 710049, China \\ ${ }^{2}$ College of Science, China Jiliang University, Hangzhou 310018, China \\ Correspondence should be addressed to Fang Qingxiang; fangqx@cjlu.edu.cn
}

Received 24 November 2012; Accepted 6 February 2013

Academic Editor: Cesar Cruz-Hernandez

Copyright (c) 2013 Fang Qingxiang et al. This is an open access article distributed under the Creative Commons Attribution License, which permits unrestricted use, distribution, and reproduction in any medium, provided the original work is properly cited.

\begin{abstract}
The synchronization and control problem of linearly coupled singular systems is investigated. The uncoupled dynamical behavior at each node is general and can be chaotic or, otherwise the coupling matrix is not assumed to be symmetrical. Some sufficient conditions for globally exponential synchronization are derived based on Lyapunov stability theory. These criteria, which are in terms of linear matrix inequality (LMI), indicate that the left and right eigenvectors corresponding to eigenvalue zero of the coupling matrix play key roles in the stability analysis of the synchronization manifold. The controllers are designed for state feedback control and pinning control, respectively. Finally, a numerical example is provided to illustrate the effectiveness of the proposed conditions.
\end{abstract}

\section{Introduction}

Many natural and synthetic systems, such as neural systems, social systems, WWW, food webs, and electrical power grids, can all be described by complex networks. For decades, complex networks have been focused on by scientists from various fields, for instance, sociology, biology, mathematics, and physics.

Linear coupled ordinary differential equations (LCODEs) are a large class of dynamical systems with continuous time and state, as well as discrete space, for describing coupled oscillators [1]. The LCODE model is widely used to describe the models in nature and engineering. Dhamala et al. [2] study spike-burst neural activity and the transitions to a synchronized state using a model of linearly coupled bursting neurons. Lloyd and Jansen [3] present an extension of an analysis technique for an ecologically motivated spatial meta population model. Perez-Munuzuri et al. [4] study the dynamics of linearly coupled Chua circuits with application to image processing and many other cases. Many complicated dynamical behaviors of coupled chaotic oscillators have been widely studied [5-10]. Among them, the synchronization of LCODEs has been an active area for decades [11].
Synchronization is very universal in daily life. In 1665 , Huygens found the synchronous wing of two pendulums [12]. In 1680, Kempfer found fireflies' synchronized flashing. In 2000, Néda et al. [13] elaborate the mechanism of synchronized clapping in concert halls. There are countless heart cells synchronously oscillating in our hearts. Some researches indicate that thousands of Routers may synchronously send messages on the internet which will trigger network congestion. Now, the synchronization of several systems plays an important role in nuclear magnetic resonance spectrometers, signal generators, laser equipments, superconducting materials, and communication systems. The synchronization technique for LCODEs is applied in many fields. The autoprinciples for parallel image processing are presented in $[4,14,15]$. Lu and Chen [16] apply synchronization technique to recognize an image with strong robustness in recognition. The different architectures of the coupled chaotic system for communication are presented in [16-19] and many other papers. Hoppensteadt and Izhikevich [20] propose an architecture of coupled neural networks to store and retrieve complex oscillatory patterns as synchronization states. Therefore, study of the synchronization of the LCODEs is an important and necessary step for both understanding 
the dynamics of complex networks and practical design of coupled oscillators.

Recently, synchronization of LCODEs has attracted much attention from researchers in different fields. Wu and Chua [21] define a distance between the state of the coupled system and the synchronization manifold and propose a methodology for discussing global convergence for the complete regular coupling configuration. Nijmeijer and Mareels [22] view the problem of synchronization as a special case of the observer design problem and provide a reasonable comprehensive framework for synchronization issues. In $[8,23]$, the local stability of the synchronization manifold is studied via a linearization method named the "master stability function" method. Wang and Chen $[8,9]$ study synchronization of randomly coupled networks such as small-world lattices and scale-free networks. Lu and Chen [24] investigate the stability of the synchronization manifold on the basis of geometrical analysis of the synchronization manifold in which the left and right eigenvectors corresponding to eigenvalue zero of the coupling matrix play key roles. Liu et al. [25] show that the $L_{2}$ norm of the synchronization error vector presents a suitable measure of both swiftness and vibration of network synchronization and design an LQR controller to drive the network onto some homogeneous stationary states and minimize the $L_{2}$ norm of the output of the linearized network. $\mathrm{Lu}$ and $\mathrm{Ho}$ [26] distill a quantity convenient to calculate even for large-scale networks from the coupling matrix to characterize the synchronizability of the corresponding dynamical networks and obtain some criteria to guarantee the globally exponential synchronization. Liu et al. [27] propose a synchronization strategy of adjusting adaptively a node's coupling strength based on the node's local generalized synchronization information. Fan et al. [28] propose a new scheme for synchronization between two or more complex networks using scalar signals under pinning control. Guan et al. [29] introduce the concept of control topology and design an impulsive controller to achieve the exponential synchronization of complex networks with system delay and multiple coupling delays.

It is worthwhile to mention that we usually have to consider some algebraic constraints of complex networks in modeling the real-world problems. For instance, communication resources are always limited and required to be allocated to different levels of privileged users. Hence, special constraints are needed in the resource allocation process. Constructing a complex networks model with a set of constraints is necessary and indispensable. For this purpose, Xiong et al. [30] introduce linearly coupled singular systems to describe complex networks with a special class of constraints. Singular systems are very complicated since one does not know whether the solution exists, not to mention the stability or synchronization of the solution for the system regularity and impulse elimination. In fact, singular systems give a more general description of physical systems than the normal one, and many studies have extended concepts and results from the normal systems theory into the realm of singular systems [26-44]. In [30], the synchronization problem of linearly coupled singular systems with the coupling matrix assumed to be symmetrical and irreducible is investigated, and a sufficient condition for globally asymptotic synchronization is derived based on the Lyapunov stability theory.

In this paper, we further investigate the synchronization problem of linearly coupled singular systems. The model considered here is general. At each node, the uncoupled system defined by $E \dot{x}=A x+f(x, t)$ can have various dynamical behaviors. As for the coupling matrix, we do not assume symmetry. We find out that even though the synchronization manifold can be stable, the individual state may be unstable. We construct a reference state making use of the left eigenvector corresponding to eigenvalue zero of the coupling matrix and obtain criteria for globally exponential synchronization based on the Lyapunov stability theory. We design the controllers for state feedback control and pinning control. The conditions presented in the paper are based on strict linear matrix inequalities (LMIs) and can be solved directly by LMI toolbox of Matlab.

The remainder of the paper is organized as follows. In Section 2, the criteria for synchronization of the linearly coupled singular systems are obtained. In Section 3, We investigate the state feedback control and pinning control problem for synchronization of the linearly coupled singular systems. We show a simulation example to demonstrate the effectiveness of our results in Section 4. This paper is concluded in Section 5.

Notation. $\operatorname{Lip}(L)$ denotes the class of Lipschitz continuous function with Lipschitz constant $L$. $I_{n}$ stands for the $n \times n$ identity matrix. The superscript $T$ and $*$ represent transpose and conjugate transpose, respectively. For a square matrix $A$, if $A$ has only real eigenvalues, then $\lambda_{M}(A)$ denote the maximal eigenvalues of $A$. For real symmetric matrices $A$ and $B, A>B(A \geq B)$ means that $A-B$ is positive definite (or positive semidefinite). The matrices, if not explicitly stated, are assumed to have compatible dimensions.

\section{Synchronization of Linearly Coupled Singular Systems}

Consider the following linearly coupled network:

$$
\begin{aligned}
E \dot{x}_{i}(t)= & A x_{i}(t)+f\left(x_{i}(t), t\right) \\
& +c \sum_{j=1}^{N} b_{i j} \Gamma x_{j}(t), \quad i=1,2, \ldots, N,
\end{aligned}
$$

where $x_{i}(t)=\left[x_{i 1}(t), x_{i 2}(t), \ldots, x_{i n}(t)\right]^{T} \in R^{n}$ is state variable of node $i, i=1,2, \ldots, N$, and $f: R^{n} \times R \rightarrow R^{n}$ is a time-varying nonlinear function. $E, A \in R^{n \times n}$ are constant matrices; $0<\operatorname{rank}(E)=r<n$. The scalar $c>0$ is the coupling strength. $b_{i j} \geq 0$ denotes the coupling coefficient from node $j$ to node $i$, for $i, j=1, \ldots, N, i \neq j . b_{i i}=-\sum_{j=1, j \neq i}^{N} b_{i j}, i=$ $1,2, \ldots, N . \Gamma=\operatorname{diag}\left(\gamma_{1}, \ldots, \gamma_{n}\right)$ denotes the inner connection at each node with $\gamma_{i} \geq 0, i=1, \ldots, n$, which means that two nodes are connected by their $i$ th component if $\gamma_{i}>0$.

Definition 1 (see [24]). The coupled system (1) is globally exponentially synchronized if there exist $\alpha>0, t_{0}>0$, and 
$M>0$, such that for each solution $x_{i}(t), i=1,2, \ldots, N$, $\left\|x_{i}(t)-x_{j}(t)\right\| \leq M e^{-\alpha t}$ holds for all $t>t_{0}, i, j=1,2, \ldots, N$.

The objective of this section is to find the conditions ensuring the coupled system (1) globally exponentially synchronized.

Denote that $B=\left(b_{i j}\right)_{N \times N}$. The following lemma characterizes the right and left eigenvectors of $B$ corresponding to eigenvalue 0 , which play key roles in the investigation of the synchronization of the coupled system (1).

Lemma 2 (see [24]). If B is irreducible, then the following are valid.

(1) B has an eigenvalue 0 with multiplicity 1 and the right eigenvector $[1,1, \ldots, 1]^{T}$.

(2) If $\lambda$ is an eigenvalue of $B$ and $\lambda \neq 0$, then $\operatorname{Re}(\lambda)<0$.

(3) Suppose that $\xi=\left[\xi_{1}, \xi_{2}, \ldots, \xi_{N}\right]^{T} \in R^{N}$ (without loss of generality, assume $\sum_{i=1}^{N} \xi_{i}=1$ ) is the left eigenvector of $B$ corresponding to eigenvalue 0 ; then, $\xi_{i}>0$ holds for all $i=1,2, \ldots, N$.

In the following discussion, we always assume that $B$ is irreducible and $f(x, t) \in \operatorname{Lip}(L)$.

Let $\hat{x}(t)=\sum_{i=1}^{N} \xi_{i} x_{i}(t), e_{i}(t)=x_{i}(t)-\widehat{x}(t), f_{i}(x, t)=$ $f\left(x_{i}, t\right)-\sum_{k=1}^{N} \xi_{k} f\left(x_{k}(t), t\right), i=1,2, \ldots, N$. Noticing that $\sum_{j=1}^{N} b_{i j}=0, i=1,2, \ldots, N$ and $\sum_{i=1}^{N} \xi_{i} b_{i j}=0, j=1,2, \ldots, N$, we have

$$
E \dot{e}_{i}(t)=A e_{i}(t)+c \sum_{j=1}^{N} b_{i j} \Gamma e_{j}(t)+f_{i}(x, t), \quad i=1,2, \ldots, N .
$$

Denote that $e(t)=\left[e_{1}(t), \ldots, e_{N}(t)\right]$, and $F(t)=\left[f_{1}(x, t)\right.$, $\left.\ldots, f_{N}(x, t)\right]$; then,

$$
E \dot{e}(t)=A e(t)+c \Gamma e(t) B^{T}+F(x, t) .
$$

Let $B^{T}=W J W^{-1}$ be the Jordan decomposition of $B^{T}$, where $J=\operatorname{diag}\left(J_{1}, J_{2}, \ldots, J_{l}\right), J_{j}$ is $\sigma_{j}$-order Jordan block, $j=1,2, \ldots, l$, and $\sum_{j=1}^{l} \sigma_{j}=N$. Denote the diagonal element of $J_{j}$ by $\lambda_{j}, j=1,2, \ldots, l$. Without loss of generality, we assume that $\lambda_{1}=0$, and then $J_{1}=0$ is a scalar.

Define

$$
y(t)=\left[y_{1}(t), y_{2}(t), \ldots, y_{N}(t)\right]=e(t) W,
$$

and denote that $W=\left[W_{1}, W_{2}, \ldots, W_{N}\right]=\left(w_{i j}\right)_{N \times N}$; then, $y_{i}(t)=e(t) W_{i}, i=1,2, \ldots, N$.

Denote that $\rho_{0}=0$ and $\rho_{j}=\sum_{k=1}^{j} \sigma_{k}, j=1,2, \ldots, l$; then, we can rewrite the coupled system (3) as

$$
\begin{gathered}
E \dot{y}_{i}(t)=\left(A+c \lambda_{k} \Gamma\right) y_{i}(t)+F(x, t) W_{i}, \\
i=\rho_{k-1}+1, k=1,2, \ldots, l, \\
E \dot{y}_{i}(t)=\left(A+c \lambda_{k} \Gamma\right) y_{i}(t)+c \Gamma y_{i-1}(t)+F(x, t) W_{i}, \\
\rho_{k-1}+1<i<\rho_{k}, k=2, \ldots, l .
\end{gathered}
$$

For $j=1,2, \ldots, l$, define

$$
\begin{aligned}
& z_{j}=\left[\begin{array}{c}
y_{\rho_{j-1}+1} \\
y_{\rho_{j-1}+2} \\
\vdots \\
y_{\rho_{j}}
\end{array}\right] \text {, } \\
& A_{j}=\left[\begin{array}{cccc}
A+c \lambda_{j} \Gamma & & & \\
c \Gamma & A+c \lambda_{j} \Gamma & & \\
& \ddots & \ddots & \\
& & c \Gamma & A+c \lambda_{j} \Gamma
\end{array}\right] \text {, } \\
& E_{j}=\left[\begin{array}{llll}
E & & & \\
& E & & \\
& & \ddots & \\
& & & E
\end{array}\right]_{\left(\sigma_{j} n\right) \times\left(\sigma_{j} n\right)} \text {, } \\
& F_{j}(x, t)=\left[\begin{array}{c}
F(x, t) W_{\rho_{j-1}+1} \\
F(x, t) W_{\rho_{j-1}+2} \\
\vdots \\
F(x, t) W_{\rho_{j}}
\end{array}\right] \text {, }
\end{aligned}
$$

and then the coupled system (5) can be rewritten as

$$
E_{j} \dot{z}_{j}(t)=A_{j} z_{j}(t)+F_{j}(x, t), \quad j=1,2, \ldots, l .
$$

Let $z(t)=\left[z_{1}^{T}(t), z_{2}^{T}(t), \ldots, z_{l}^{T}(t)\right]^{T}$; then, with the previous decompositions, synchronization of the coupled system (1) is equivalent to $z(t) \rightarrow 0$. In the following, instead of investigating $x(t)$, we investigate dynamical behaviors of $z(t)$ directly.

For the sake of convenient expression, we give the following definition.

Definition 3 (see $[45,46])$. The pair $(E, A)$ is said to be regular if $\operatorname{det}(s E-A)$ is not identical zero. The pair $(E, A)$ is said to be impulse-free if $\operatorname{deg}(\operatorname{det}(s E-A))=\operatorname{rank}(E)$.

Suppose that $W^{-1}=\left[\widetilde{W}_{1}, \ldots, \widetilde{W}_{N}\right]=\left(\widetilde{w}_{i j}\right)_{N \times N}$. For $j=$ $2, \ldots, l, k=1,2, \ldots, N, s=1,2, \ldots, N$, define

$$
\begin{aligned}
& \alpha_{j}=\sum_{d=1}^{\sigma_{j}}\left(\sum_{i=1}^{N}\left|w_{i, \rho_{j-1}+d}\right|\right)^{2}, \\
& \beta_{k j}=\sum_{d=1}^{\sigma_{j}}\left(\left|w_{k, \rho_{j-1}+d}\right| \sum_{i=1}^{N}\left|w_{i, \rho_{j-1}+d}\right|\right), \\
& \gamma_{k j}=\alpha_{j} \xi_{k}+\beta_{k j}, \quad \phi_{k s}=\sum_{j=2 i=1}^{l} \sum_{i=1}^{N} \gamma_{i j} \widetilde{w}_{k i}^{*} \widetilde{w}_{s i},
\end{aligned}
$$

and denote

$$
\phi=\left[\begin{array}{cccc}
\phi_{22} & \phi_{23} & \cdots & \phi_{2 N} \\
\phi_{32} & \phi_{33} & \cdots & \phi_{3 N} \\
\vdots & \vdots & \ddots & \vdots \\
\phi_{N 2} & \phi_{N 3} & \cdots & \phi_{N N}
\end{array}\right], \quad \Phi=\phi \otimes I_{n} .
$$


In the following, we give the main conclusion of this section.

Theorem 4. If there exists a scalar $\varepsilon>0$ and matrices $P_{j} \in$ $R^{\left(\sigma_{j} n\right) \times\left(\sigma_{j} n\right)}, j=2,3, \ldots, l$, satisfying

$$
\begin{gathered}
E_{j}^{T} P_{j}=P_{j}^{T} E_{j} \geq 0, \quad j=2,3, \ldots, l \\
\widehat{P}+2 L^{2} \Phi+\varepsilon I_{(N-1) n}<0
\end{gathered}
$$

where $\widehat{P}=\operatorname{diag}\left(P_{2}^{T} P_{2}+A_{2}^{*} P_{2}+P_{2}^{T} A_{2}, \ldots, P_{l}^{T} P_{l}+A_{l}^{*} P_{l}+\right.$ $\left.P_{l}^{T} A_{l}\right)$, then the coupled system (1) is globally exponentially synchronized.

Proof. First, note that $\lambda_{1}=0$ and corresponding left eigenvector $\xi$ satisfies that $\sum_{i=1}^{N} \xi_{i}=1$. This corresponds to synchronization on the manifold $z_{1}(t)=0$.

Next, we consider the manifolds $z_{2}(t)=0, z_{3}(t)=$ $0, \ldots, z_{l}(t)=0$.
Choose the Lyapunov function candidate as follows:

$$
V(t)=\frac{1}{2} \sum_{j=2}^{l} z_{j}^{*}(t) P_{j}^{T} E_{j} z_{j}(t),
$$

and then the derivative of $V(t)$ along the trajectory of (7) yields

$\dot{V}(t)$

$$
\begin{aligned}
& =\frac{1}{2} \sum_{j=2}^{l}\left(z_{j}^{*}\left(A_{j}^{*} P_{j}+P_{j}^{T} A_{j}\right) z_{j}+F_{j}^{*}(x, t) P_{j} z_{j}+z_{j}^{*} P_{j}^{T} F_{j}(x, t)\right) \\
& \leq \frac{1}{2} \sum_{j=2}^{l}\left(z_{j}^{*}\left(P_{j}^{T} P_{j}+A_{j}^{*} P_{j}+P_{j}^{T} A_{j}\right) z_{j}+F_{j}^{*}(x, t) F_{j}(x, t)\right) .
\end{aligned}
$$
and

Since $e_{i}(t)=y(t) \widetilde{W}_{i}, i=1,2, \ldots, N, f(x, t) \in \operatorname{Lip}(L)$, we have

$$
\begin{aligned}
& F_{j}^{*}(x, t) F_{j}(x, t)=\sum_{d=1}^{\sigma_{j}} \sum_{k=1}^{N} \sum_{s=1}^{N} w_{k, \rho_{j-1}+d}^{*} w_{s, \rho_{j-1}+d} \\
& \times\left[\left(f^{*}\left(x_{k}, t\right)-f^{*}(\widehat{x}, t)\right)\right. \\
& \times\left(f\left(x_{s}, t\right)-f(\widehat{x}, t)\right) \\
& +2 \sum_{v=1}^{N} \xi_{v}\left(f^{*}\left(x_{k}, t\right)-f^{*}(\widehat{x}, t)\right) \\
& \times\left(f\left(x_{v}, t\right)-f(\widehat{x}, t)\right) \\
& +\sum_{v=1}^{N} \sum_{i=1}^{N} \xi_{v} \xi_{i}\left(f^{*}\left(x_{v}, t\right)-f^{*}(\widehat{x}, t)\right) \\
& \left.\times\left(f\left(x_{i}, t\right)-f(\widehat{x}, t)\right)\right] \\
& \leq 2 \sum_{d=1}^{\sigma_{j}}\left(\sum_{s=1}^{N}\left|w_{s, \rho_{j-1}+d}\right|\right) \\
& \times \sum_{k=1}^{N}\left|w_{k, \rho_{j-1}+d}\right|\left\|f\left(x_{k}, t\right)-f(\widehat{x}, t)\right\|^{2}
\end{aligned}
$$

$$
\begin{array}{r}
+2\left(\sum_{d=1}^{\sigma_{j}}\left(\sum_{i=1}^{N}\left|w_{i, \rho_{j-1}+d}\right|\right)^{2}\right) \\
\times \sum_{v=1}^{N} \xi_{v}\left\|f\left(x_{v}, t\right)-f(\widehat{x}, t)\right\|^{2} \\
\leq 2 L^{2} \sum_{k=1}^{N} \gamma_{k j} e_{k}^{*} e_{k} \\
=2 L^{2} \sum_{k=1}^{N} \gamma_{k j} \widetilde{W}_{k}^{*} y^{*}(t) y(t) \widetilde{W}_{k} \\
=2 L^{2} \sum_{v=2}^{N} \sum_{s=2}^{N}\left(\sum_{k=1}^{N} \gamma_{k j} \widetilde{w}_{v k}^{*} \widetilde{w}_{s k}\right) y_{v}^{*} y_{s},
\end{array}
$$

$\dot{V}(t)$$$
\leq \frac{1}{2}\left(\sum_{j=2}^{l} z_{j}^{*}\left(P_{j}^{T} P_{j}+A_{j}^{*} P_{j}+P_{j}^{T} A_{j}\right) z_{j}\right.
$$$$
\left.+2 L^{2} \sum_{k=2 s=2}^{N} \sum_{k s}^{N} \phi_{k} y_{s}\right)
$$ 
that is,

$$
\dot{V}(t) \leq \frac{1}{2} \widehat{z}^{*}(t)\left(\widehat{P}+2 L^{2} \Phi\right) \widehat{z}(t),
$$

where $\widehat{z}(t)=\left[z_{2}^{T}(t), \ldots, z_{l}^{T}(t)\right]^{T}$.

According to (11), one has

$$
\dot{V}(t)<-\frac{1}{2} \varepsilon \sum_{j=2}^{N} z_{j}^{*} z_{i} \leq-\frac{\varepsilon}{\kappa} V(t),
$$

where $\kappa=\max \left\{\lambda_{M}\left(P_{j}^{T} E_{j}\right), j=2,3, \ldots, l\right\}$.

Denote that

$$
\widehat{W}=\left[\begin{array}{cccc}
\widetilde{w}_{21} & \widetilde{w}_{22} & \cdots & \widetilde{w}_{2 N} \\
\widetilde{w}_{31} & \widetilde{w}_{32} & \cdots & \widetilde{w}_{3 N} \\
\vdots & \vdots & \ddots & \vdots \\
\widetilde{w}_{N 1} & \widetilde{w}_{N 2} & \cdots & \widetilde{w}_{N N}
\end{array}\right]
$$

$\widehat{\gamma}_{i}=\sum_{j=2}^{l} \gamma_{i j}, i=1,2, \ldots, N$, and $\widehat{\Gamma}=\operatorname{diag}\left(\widehat{\gamma}_{1}, \ldots, \widehat{\gamma}_{N}\right)$; then, $\phi=\left(\widehat{W}^{T}\right)^{*} \widehat{\Gamma}^{T}$, which implies that $\phi$ and $\Phi$ are both positive definite matrices.

It follows from (10) and (11) that the pairs $\left(E_{j}, A_{j}\right), j=$ $2, \ldots, l$, are all regular and impulse-free [45]; that is, there exist nonsingular matrices $M_{j}, Q_{j} \in C^{\left(\sigma_{j} n\right) \times\left(\sigma_{j} n\right)}$ satisfying that

$$
\begin{gathered}
M_{j} E_{j} Q_{j}=\operatorname{diag}\left(I_{\sigma_{j} r}, 0\right), \\
M_{j} A_{j} Q_{j}=\operatorname{diag}\left(\widetilde{A}_{j}, I_{\sigma_{j}(n-r)}\right),
\end{gathered}
$$

for $j=2,3, \ldots, N$.

By (10) and (11), we can also obtain that $\left(M_{j}^{-1}\right)^{*} P_{j} Q_{j}=$ $\left[\begin{array}{cc}P_{j 1} & 0 \\ P_{j 3} & P_{j 4}\end{array}\right]$, where $P_{j 1} \in R^{\left(\sigma_{j} r\right) \times\left(\sigma_{j} r\right)}$ and $P_{j 1}>0, j=2,3, \ldots, N$ [36].

Let $Q_{j}^{-1} z_{j}=\left[z_{j 1}^{T}, z_{j 2}^{T}\right]^{T}$ and $M_{j} F_{j}(x, t)=\left[\left(F_{j 1}(x, t)\right)^{T}\right.$, $\left.\left(F_{j 1}(x, t)\right)^{T}\right]^{T}$, where $z_{j 1} \in C^{\sigma_{j} r}$ and $F_{j 1}(x, t) \in C^{\sigma_{j} r}, j=$ $1,2, \ldots, l$, and then the system (7) can be rewritten as

$$
\begin{gathered}
\dot{z}_{j 1}(t)=\widetilde{A}_{j} z_{j 1}(t)+F_{j 1}(x, t), \quad j=1,2, \ldots, l, \\
z_{j 2}(t)=-F_{j 2}(x, t), \\
V(t)=\frac{1}{2} \sum_{j=2}^{l} z_{j 1}^{*} P_{j 1} z_{j 1} .
\end{gathered}
$$

Since the matrix $P_{j 1}$ is positive definite, $z_{j 1}(t), i=2,3, \ldots, l$, are globally exponentially stable. In the following, we show that $z_{j 2}(t), j=2,3, \ldots, l$, are also globally exponentially stable; thus, the coupled system (1) is globally exponentially synchronized.

Denote that $M_{j}=\left[\begin{array}{ll}M_{j 1}^{T} & M_{j 2}^{T}\end{array}\right]^{T}$, where $M_{j 1} \in C^{\left(\sigma_{j} r\right) \times\left(\sigma_{j} n\right)}$, $j=1,2, \ldots, l$. Without loss of generality, we assume that $\left\|M_{j 2}\right\|=1[40]$.
From (20), we have

$$
\begin{aligned}
\left\|z_{j 2}(t)\right\|^{2} & \leq\left\|M_{j 2}\right\|^{2}\left\|F_{j}(x, t)\right\|^{2}=F_{j}^{*}(x, t) F_{j}(x, t) \\
& \leq 2 L^{2} \sum_{v=2}^{N} \sum_{s=2}^{N} \eta_{j v s} y_{v}^{*} y_{s},
\end{aligned}
$$

where $\eta_{j v s}=\sum_{k=1}^{N} \gamma_{k j} \widetilde{w}_{v k}^{*} \widetilde{w}_{s k}, j=2,3, \ldots, l, v=1,2, \ldots, N$, $s=1,2, \ldots, N$.

For $j=2,3, \ldots, l$, define

$$
\Psi_{j}=\left[\begin{array}{cccc}
\eta_{j 22} I_{n} & \eta_{j 23} I_{n} & \cdots & \eta_{j 2 N} I_{n} \\
\eta_{j 32} I_{n} & \eta_{j 33} I_{n} & \cdots & \eta_{j 3 N} I_{n} \\
\vdots & \vdots & \ddots & \vdots \\
\eta_{j N 2} I_{n} & \eta_{j N 3} I_{n} & \cdots & \eta_{j N N} I_{n}
\end{array}\right],
$$

and let $\lambda_{\Psi_{j}}$ stand for the maximal eigenvalue of the matrix $\Psi_{j}$; then,

$$
\begin{aligned}
\left\|z_{j 2}(t)\right\|^{2} \leq & 2 L^{2} \lambda_{\Psi_{j}} \lambda_{Q} \\
& \times \sum_{k=2}^{l}\left(\left\|z_{k 1}(t)\right\|^{2}+\left\|z_{k 2}(t)\right\|^{2}\right), \quad j=2,3, \ldots, l,
\end{aligned}
$$

$$
\sum_{j=2}^{l}\left\|z_{j 2}(t)\right\|^{2} \leq 2 L^{2} \lambda_{\mathrm{Q}} \lambda_{\Psi} \sum_{k=2}^{l}\left(\left\|z_{k 1}(t)\right\|^{2}+\left\|z_{k 2}(t)\right\|^{2}\right),
$$

where $\lambda_{\mathrm{Q}}$ is the maximal eigenvalue of the matrix $\operatorname{diag}\left(Q_{2}^{*} Q_{2}\right.$, $\left.Q_{3}^{*} Q_{3}, \ldots, Q_{l}^{*} Q_{l}\right)$ and $\lambda_{\Psi}=\sum_{j=2}^{l} \lambda_{\Psi_{j}}$.

From (25), we have

$$
\left(1-2 L^{2} \lambda_{\mathrm{Q}} \lambda_{\Psi}\right) \sum_{j=2}^{l}\left\|z_{j 2}(t)\right\|^{2} \leq 2 L^{2} \lambda_{\mathrm{Q}} \lambda_{\Psi} \sum_{k=2}^{l}\left\|z_{k 1}(t)\right\|^{2} .
$$

By choosing suitably $Q_{2}, Q_{3}, \ldots, Q_{l}$ such that $1-2 L^{2} \lambda_{Q} \lambda_{\Psi}>$ 0 , we have

$$
\sum_{j=2}^{l}\left\|z_{j 2}(t)\right\|^{2} \leq \frac{2 L^{2} \lambda_{\mathrm{Q}} \lambda_{\Psi}}{1-2 L^{2} \lambda_{\mathrm{Q}} \lambda_{\Psi}} \sum_{k=2}^{l}\left\|z_{k 1}(t)\right\|^{2}
$$

consequently, $z_{j 2}(t), j=2,3, \ldots, N$, are globally exponentially stable.

The following lemma is the combination of Theorem 4 and Lemma 2 of [33].

Lemma 5 (see [33]). Suppose that $E, A \in R^{n \times n}, B \in R^{n \times q}$, $C \in R^{p \times n}, \gamma>0$ is a scalar, $0<\operatorname{rank}(E)=r<n, E=$ $E_{L} E_{R}^{T}, E_{L}, E_{R} \in R^{n \times r}$ are of full column rank, $U, V \in R^{n \times(n-r)}$ are of full column rank and composed of bases of $\operatorname{Null}(E)$ and $\operatorname{Null}\left(E^{T}\right)$, respectively. There exists $P \in R^{n \times n}$ satisfying

$$
P^{T} A+A^{T} P+B B^{T}+\frac{P^{T} C^{T} C P}{\gamma^{2}}<0, \quad E^{T} P=P^{T} E \geq 0,
$$


if and only if there exist a symmetric matrix $Y \in R^{n \times n}$ and a matrix $S \in R^{(n-r) \times(n-r)}$ satisfying

$$
\left[\begin{array}{cc}
\Phi_{1} & \Phi_{2} \\
\Phi_{2}^{T} & -\gamma^{2} I
\end{array}\right]<0, \quad E_{L}^{T} Y E_{L}>0
$$

where $\Phi_{1}=A^{T} S_{1}+S_{1}^{T} A+B B^{T}, \Phi_{2}=S_{1}^{T} C^{T}$, and $S_{1}=Y E+$ $V S U^{T}$.

Let $\lambda_{\Phi}$ be the maximal eigenvalue of the matrix $\Phi, E_{j}=$ $E_{j L} E_{j R}^{T}$, where $E_{j L}, E_{j R} \in R^{\left(\sigma_{j} n\right) \times\left(\sigma_{j} r\right)}$ are of full column rank, and $U_{j}, V_{j} \in R^{\left(\sigma_{j} n\right) \times\left(\sigma_{j}(n-r)\right)}$ are of full column rank and composed of bases of $\operatorname{Null}\left(E_{j}\right)$ and $\operatorname{Null}\left(E_{j}^{T}\right)$, respectively, $j=2,3, \ldots, l$.

Based on Theorem 4 and Lemma 5, we have the following conclusion.

Theorem 6. If there exist a scalar $\varepsilon>0$, symmetric matrices $Y_{j} \in R^{\left(\sigma_{j} n\right) \times\left(\sigma_{j} n\right)}$, and matrices $S_{j} \in R^{\left(\sigma_{j}(n-r)\right) \times\left(\sigma_{j}(n-r)\right)}$ satisfying

$$
\left[\begin{array}{cc}
\left(2 L^{2} \lambda_{\Phi}+\varepsilon\right) I_{\sigma_{j} n}-A_{j}^{*} A_{j} & A_{j}^{*}+\Lambda_{j}^{T} \\
A_{j}+\Lambda_{j} & -I_{\sigma_{j} n}
\end{array}\right]<0, \quad E_{j L}^{T} Y_{j} E_{j L}>0,
$$

where $\Lambda_{j}=Y_{j} E_{j}+V_{j} S_{j} U_{j}^{T}, j=2,3, \ldots, l$, then the coupled system (1) is globally exponentially synchronized.

When $B$ is a real symmetric matrix, $J=\operatorname{diag}\left(\lambda_{1}, \lambda_{2}, \ldots\right.$, $\left.\lambda_{N}\right)$ and $W$ is an orthogonal matrix, where $0=\lambda_{1} \geq \lambda_{2} \geq$ $\cdots \geq \lambda_{N}$ are the eigenvalues of $B$. The coupled system (3) can be rewritten as

$$
E \dot{y}_{i}(t)=\left(A+c \lambda_{i} \Gamma\right) y_{i}(t)+F(x, t) W_{i}, \quad i=1,2, \ldots, N
$$

For $j=2, \ldots, N, k=1,2, \ldots, N$, and $v=1,2, \ldots, N$, define

$$
\begin{aligned}
& \alpha_{s j}=\left(\sum_{i=1}^{N}\left|w_{i j}\right|\right)^{2}, \quad \beta_{s k j}=\left|w_{k j}\right| \sum_{i=1}^{N}\left|w_{i j}\right|, \\
& \gamma_{s k j}=\alpha_{j} \xi_{k}+\beta_{k j}, \quad \phi_{s k v}=\sum_{j=2}^{N} \sum_{i=1}^{N} \gamma_{s i j} \widetilde{w}_{k i}^{*} \widetilde{w}_{v i} \\
& \phi_{s}=\left[\begin{array}{cccc}
\phi_{s 22} & \phi_{s 23} & \cdots & \phi_{s 2 N} \\
\phi_{s 32} & \phi_{s 33} & \cdots & \phi_{s 3 N} \\
\vdots & \vdots & \ddots & \vdots \\
\phi_{s N 2} & \phi_{s N 3} & \cdots & \phi_{s N N}
\end{array}\right], \quad \Phi_{s}=\phi_{s} \otimes I_{n},
\end{aligned}
$$

and denote that $\lambda_{\Phi_{s}}$ is the maximal eigenvalue of $\Phi_{s}, E=$ $E_{L} E_{R}^{T}, E_{L}, E_{R} \in R^{n \times r}$ are of full column rank, $U, V \in R^{n \times(n-r)}$ are of full column rank and composed of bases of $\operatorname{Null}(E)$ and $\operatorname{Null}\left(E^{T}\right)$, respectively.

Similar to the proof of Theorem 6, we have the following conclusion.
Theorem 7. Suppose that $B$ is a real symmetric matrix. If there exist a scalar $\varepsilon>0$, a symmetric matrix $Y \in R^{n \times n}$ and a matrix $S \in R^{(n-r) \times(n-r)}$ satisfying

$$
\begin{gathered}
E_{L}^{T} Y E_{L}>0 \\
{\left[\begin{array}{cc}
\left(2 L^{2} \lambda_{\Phi_{s}}+\varepsilon\right) I_{n}-\left(A+c \lambda_{i} \Gamma\right)^{T}\left(A+c \lambda_{i} \Gamma\right) & \left(A+c \lambda_{i} \Gamma\right)^{T}+\Lambda^{T} \\
A+c \lambda_{i} \Gamma+\Lambda & -I_{n}
\end{array}\right]<0}
\end{gathered}
$$

where $\Lambda=Y E+V S U^{T}, i=2,3, \ldots, N$, and then the coupled system (1) is globally exponentially synchronized.

Remark 8. By Schur complement, the inequality (34) is equivalent to

$$
\left(2 L^{2} \lambda_{\Phi_{s}}+\varepsilon\right) I_{n}+\Lambda^{T} \Lambda+A^{T} \Lambda+\Lambda^{T} A+c \lambda_{i}\left(\Gamma \Lambda+\Lambda^{T} \Gamma\right)<0 .
$$

When $\Gamma \Lambda+\Lambda^{T} \Gamma>0$, if there exists a scalar $c_{1}>0$ satisfying

$$
\left(2 L^{2} \lambda_{\Phi_{s}}+\varepsilon\right) I_{n}+\Lambda^{T} \Lambda+A^{T} \Lambda+\Lambda^{T} A+c_{1} \lambda_{2}\left(\Gamma \Lambda+\Lambda^{T} \Gamma\right)<0,
$$

then inequality (34) is right for all $c \in\left[c_{1}, \infty\right)$. When $\Gamma \Lambda+$ $\Lambda^{T} \Gamma<0$, if there exists a scalar $c_{2}>0$ satisfying

$$
\left(2 L^{2} \lambda_{\Phi_{s}}+\varepsilon\right) I_{n}+\Lambda^{T} \Lambda+A^{T} \Lambda+\Lambda^{T} A+c_{2} \lambda_{N}\left(\Gamma \Lambda+\Lambda^{T} \Gamma\right)<0,
$$

then inequality (34) is right for all $c \in\left[0, c_{2}\right]$.

\section{Control of Linearly Coupled Singular Systems}

In this section, we investigate the state feedback control and pinning control problems of linearly coupled singular systems.

3.1. State Feedback Control. Suppose that the linearly coupled network with a state feedback controller is

$$
\begin{gathered}
E \dot{x}_{i}(t)=A x_{i}(t)+f\left(x_{i}(t), t\right)+c \sum_{j=1}^{N} b_{i j} \Gamma x_{j}(t)+D u_{i}(t), \\
u_{i}(t)=-K x_{i}(t), \quad i=1,2, \ldots, N,
\end{gathered}
$$

where $D \in R^{n \times m}$ is constant matrix, and $K \in R^{m \times n}$ is feedback gain matrix.

We continue to use the symbols in Section 2 , and let $D_{j}=$ $\operatorname{diag}(D, D, \ldots, D), K_{j}=\operatorname{diag}(K, K, \ldots, K), D_{j} \in R^{\left(\sigma_{j} n\right) \times\left(\sigma_{j} m\right)}$, $K_{j} \in R^{\left(\sigma_{j} m\right) \times\left(\sigma_{j} n\right)}, j=1,2, \ldots, l$; then, the closed loop coupled network (38) can be rewritten as

$$
E_{j} \dot{z}_{j}(t)=\left(A_{j}-D_{j} K_{j}\right) z_{j}(t)+F_{j}(x(t), t) .
$$

Follow the proof of Theorem 6, we have the following conclusion. 
Theorem 9. If there exist a scalar $\varepsilon>0$, symmetric matrices $Y_{j} \in R^{\left(\sigma_{j} n\right) \times\left(\sigma_{j} n\right)}$, matrices $S_{j} \in R^{\left(\sigma_{j}(n-r)\right) \times\left(\sigma_{j}(n-r)\right)}$, and matrix $K \in R^{m \times n}$ satisfying

$$
\begin{gathered}
E_{j L}^{T} Y_{j} E_{j L}>0, \\
{\left[\begin{array}{cc}
\left(2 L^{2} \lambda_{\Phi}+\varepsilon\right) I_{\sigma_{j} n}-A_{j}^{*} A_{j}+A_{j}^{*} D_{j} K_{j}+K_{j}^{T} D_{j} A_{j} & A_{j}^{*}-K_{j}^{T} D_{j}^{T}+\Lambda_{j}^{T} \\
A_{j}-D_{j} K_{j}+\Lambda_{j} & -I_{\sigma_{j} n}
\end{array}\right]<0,}
\end{gathered}
$$

where $\Lambda_{j}=Y_{j} E_{j}+V_{j} S_{j} U_{j}^{T}, j=1,2, \ldots, l$, then the closed loop network (39) is globally exponentially synchronized with the state feedback $u_{i}(t)=-K x_{i}(t), i=1,2, \ldots, N$.

3.2. Pinning Control. Suppose that the linearly coupled network with a single controller is

$$
\begin{gathered}
E \dot{x}_{1}(t)=A x_{1}(t)+f\left(x_{1}(t), t\right)+c \sum_{j=1}^{N} b_{1 j} \Gamma x_{j}(t)-\widetilde{K} e_{1}(t), \\
E \dot{x}_{i}(t)=A x_{i}(t)+f\left(x_{i}(t), t\right)+c \sum_{j=1}^{N} b_{i j} \Gamma x_{j}(t), \\
i=2,3, \ldots, N,
\end{gathered}
$$

where $\widetilde{K} \in R^{n \times n}$ is the feedback gain matrix.

Denote that $\widehat{E}=\operatorname{diag}\left(E_{2}, E_{3}, \ldots, E_{l}\right), \widehat{A}=\operatorname{diag}\left(A_{2}\right.$, $\left.A_{3}, \ldots, A_{l}\right), \widehat{F}(x, t)=\left[F_{2}^{T}(x, t), F_{3}^{T}(x, t), \ldots, F_{l}^{T}(x, t)\right]^{T}, \widehat{z}=$ $\left[y_{2}^{T}, y_{3}^{T}, \ldots, y_{l}^{T}\right]^{T}, \Gamma_{i}=\xi_{1}\left(\sum_{k=1}^{N} w_{k i}\right) \widetilde{K}-w_{1 i} I_{n}, i=2,3, \ldots, N$, and $\widetilde{\Gamma}=\left[\Gamma_{2}^{T}, \Gamma_{3}^{T}, \ldots, \Gamma_{N}^{T}\right]^{T}\left[\widetilde{w}_{21} I_{n}, \widetilde{w}_{31} I_{n}, \ldots, \widetilde{w}_{N 1} I_{n}\right]$; then, the coupled network (41) can be rewritten as

$$
\widehat{E} \dot{\vec{z}}=(\widehat{A}+\widetilde{\Gamma}) \widehat{z}+\widehat{F}(x, t) .
$$

Denote that

$$
\begin{aligned}
& \widetilde{\alpha}=\sum_{j=2}^{N}\left(\sum_{s=1}^{N}\left|w_{s j}\right|\right)^{2}, \\
& \widetilde{\beta}_{k}=\sum_{j=2}^{N}\left|w_{k j}\right| \sum_{s=1}^{N}\left|w_{s j}\right|, \quad \widetilde{\gamma}_{k}=\widetilde{\alpha} \xi_{k}+\widetilde{\beta}_{k}, \quad k=1,2, \ldots, N,
\end{aligned}
$$$$
\widetilde{\phi}_{v s}=\sum_{k=1}^{N} \widetilde{\gamma}_{k} \widetilde{w}_{v k}^{*} \widetilde{w}_{s k}, \quad v, s=2,3, \ldots, N,
$$$$
\widetilde{\phi}=\left[\begin{array}{cccc}
\tilde{\phi}_{22} & \tilde{\phi}_{23} & \cdots & \tilde{\phi}_{2 N} \\
\widetilde{\phi}_{32} & \widetilde{\phi}_{33} & \cdots & \widetilde{\phi}_{3 N} \\
\vdots & \vdots & \ddots & \vdots \\
\tilde{\phi}_{N 2} & \tilde{\phi}_{N 3} & \cdots & \tilde{\phi}_{N N}
\end{array}\right], \quad \widetilde{\Phi}=\widetilde{\phi} \otimes I_{n}
$$

and let $\widehat{E}=\widehat{E}_{L} \widehat{E}_{R}^{T}$, where $\widehat{E}_{L}, \widehat{E}_{R} \in R^{((N-1) n) \times((N-1) r)}$ are of full column rank, and $\widehat{U}, \widehat{V} \in R^{((N-1) n) \times((N-1)(n-r))}$ are of full column rank and composed of bases of $\operatorname{Null}(\widehat{E})$ and $\operatorname{Null}\left(\widehat{E}^{T}\right)$; then, we have the following conclusion.
Theorem 10. If there exist a scalar $\varepsilon>0$, symmetric matrices $\widehat{Y} \in R^{((N-1) n) \times((N-1) n)}$, matrices $\widehat{S} \in R^{((N-1)(n-r)) \times((N-1)(n-r))}$, and matrix $\widetilde{K} \in R^{n \times n}$ satisfying

$$
\begin{gathered}
\widehat{E}_{L}^{T} \widehat{Y} \widehat{E}_{L}>0, \\
{\left[\begin{array}{cc}
2 L^{2} \widetilde{\Phi}+\varepsilon I_{(N-1) n}-\widehat{A}^{*} \widehat{A}-\widehat{A}^{*} \widetilde{\Gamma}-\widehat{\Gamma}^{*} \widehat{A}-W_{k} & \widehat{A}^{*}+\widehat{\Gamma}^{*}+\widehat{\Lambda}^{T} \\
\widehat{A}+\widehat{\Gamma}+\widehat{\Lambda} & -I_{(N-1) n}
\end{array}\right]<0,}
\end{gathered}
$$

where $\widehat{\Lambda}=\widehat{Y} \widehat{E}+\widehat{V} \widehat{S} \widehat{U}^{T}$ and $W_{k}=\left[\widetilde{w}_{21} I_{n}, \widetilde{w}_{31} I_{n}, \ldots\right.$, $\left.\widetilde{w}_{N 1} I_{n}\right]^{*} \sum_{j=2}^{N}\left(\left|w_{1 j}\right|^{2} I_{n}-\xi_{1} w_{1 j}\left(\sum_{k=1}^{N} w_{k j}^{*}\right) \widetilde{K}^{*}-\xi_{1} w_{1 j}^{*}\left(\sum_{k=1}^{N} w_{k j}\right)\right.$ $\widetilde{K})\left[\widetilde{w}_{21} I_{n}, \widetilde{w}_{31} I_{n}, \ldots, \widetilde{w}_{N 1} I_{n}\right]$; then, the coupled network (42) is globally exponentially synchronized.

\section{A Simulation Example}

In this section, we give a numerical example to demonstrate the effectiveness of our results.

Consider linearly coupled singular systems (1), where

$$
\begin{array}{r}
x_{i}(t)=\left[x_{i 1}(t), x_{i 2}(t)\right]^{T}, f\left(x_{i}(t), t\right) \\
=\frac{1}{15}\left[\tanh \left(x_{i 1}(t)\right), \tanh \left(x_{i 2}(t)\right)\right]^{T}, \\
i=1,2, \ldots, 6,
\end{array}
$$

$$
\begin{aligned}
L & =\frac{1}{15}, \quad c=1, \quad \Gamma=I_{2}, \\
E & =\left[\begin{array}{ll}
8 & 0 \\
0 & 0
\end{array}\right], \quad A=\left[\begin{array}{cc}
0 & -3 \\
-4 & -4
\end{array}\right], \\
B & =\left[\begin{array}{cccccc}
-2 & 1 & 0 & 0 & 1 & 0 \\
1 & -5 & 1 & 1 & 1 & 1 \\
0 & 1 & -4 & 2 & 1 & 0 \\
0 & 1 & 1 & -5 & 2 & 1 \\
2 & 1 & 1 & 0 & -5 & 1 \\
2 & 1 & 1 & 0 & 1 & -5
\end{array}\right] .
\end{aligned}
$$

The Jordan canonical form of $B$ is

$$
J=\left[\begin{array}{cccccc}
0 & 0 & 0 & 0 & 0 & 0 \\
0 & -2.5325 & 0 & 0 & 0 & 0 \\
0 & 0 & -5.7338+1.0405 i & 0 & 0 & 0 \\
0 & 0 & 0 & -5.7338-1.0405 i & 1 & 0 \\
0 & 0 & 0 & 0 & -6 & 1 \\
0 & 0 & 0 & 0 & 0 & -6
\end{array}\right] \text {, }
$$

and the left eigenvector of $B$ corresponding to eigenvalue zero is

$$
\xi=[0.3508,0.1667,0.1298,0.0853,0.1809,0.0866]^{T} .
$$

Let

$$
\begin{array}{r}
e_{i}(t)=x_{i}(t)-\left[x_{1}(t), x_{2}(t), x_{3}(t), x_{4}(t), x_{5}(t), x_{6}(t)\right] \xi, \\
i=1,2, \ldots, 6,
\end{array}
$$




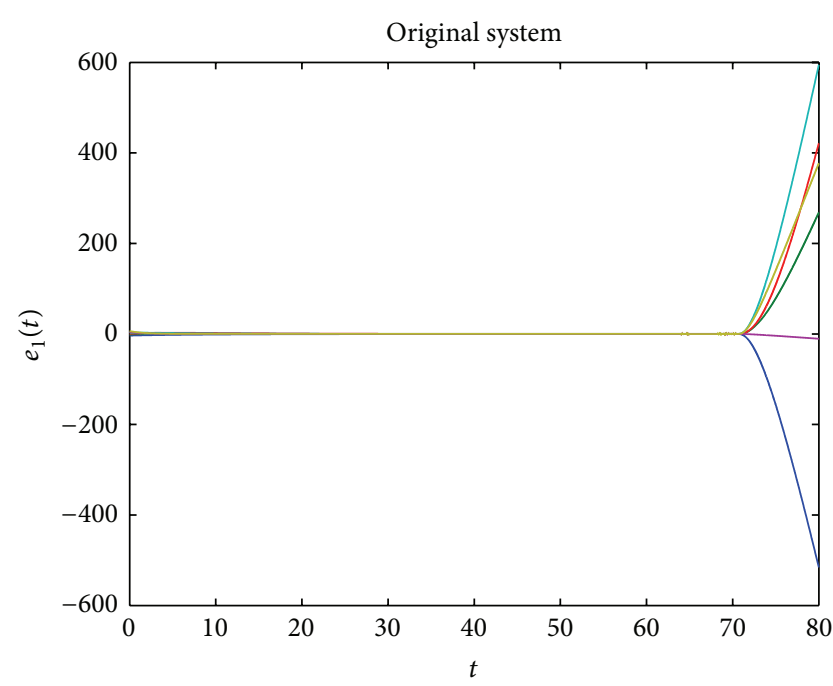

(a)

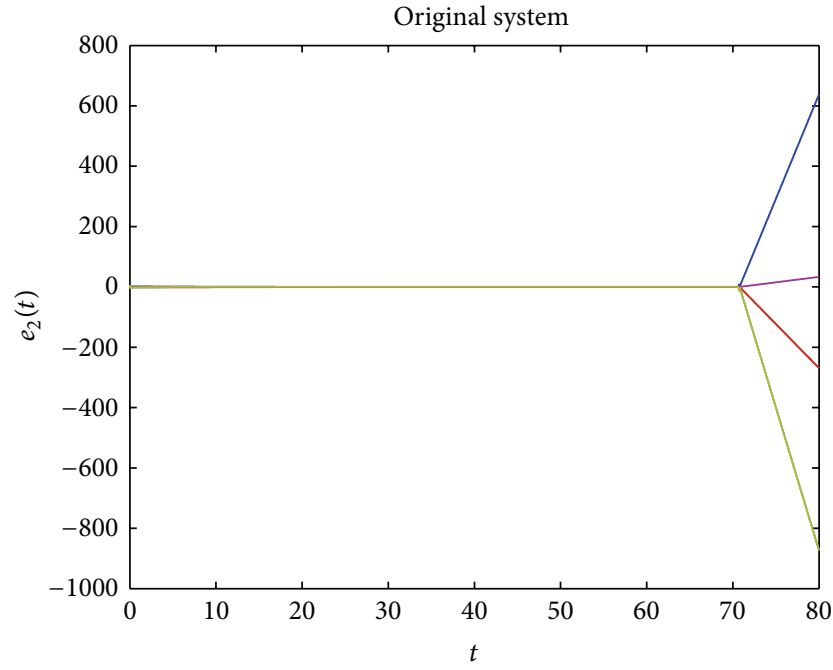

(b)

Figure 1: Curves of error variable $e_{i}(t)(i=1,2, \ldots, 6)$ of system (1).

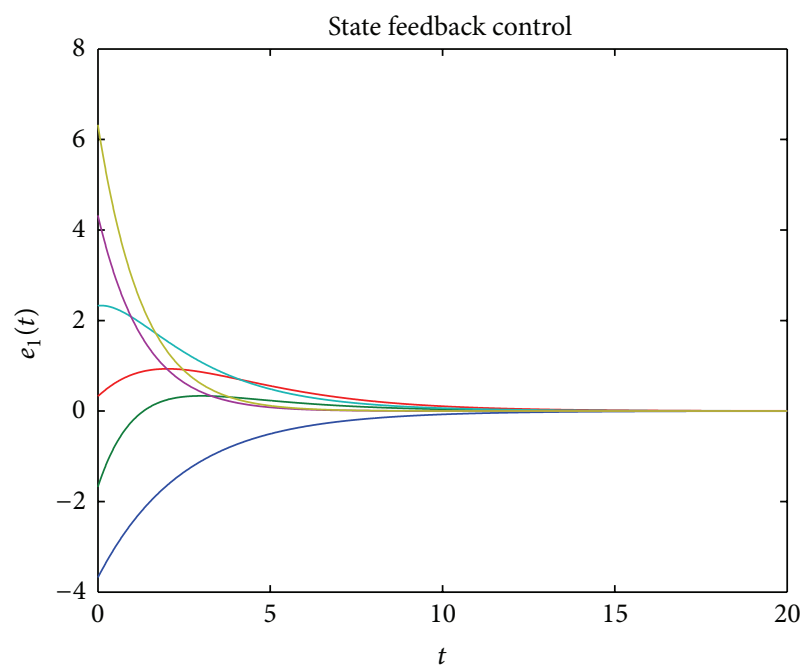

(a)

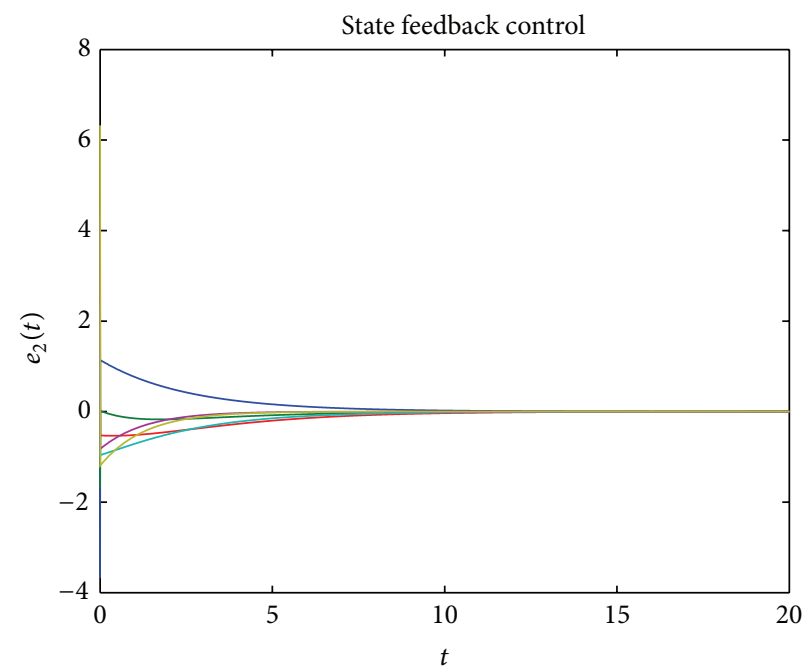

(b)

FIGURE 2: Curves of error variable $e_{i}(t)(i=1,2, \ldots, 6)$ of system (38).

and the simulation results of original system are shown in Figure 1.

From Theorem 9, the feedback gain matrix of state feedback control is

$$
K=\left[\begin{array}{cc}
0.3291 & -3.4872 \\
-2.5034 & -1.8884
\end{array}\right] \text {. }
$$

The simulation results of state feedback control are shown in Figure 2.

From Theorem 10, the feedback gain matrix of pinning control is

$$
\widetilde{K}=\left[\begin{array}{cc}
3.4992 & -2.7554 \\
-1.0090 & 1.4619
\end{array}\right] \text {. }
$$

The simulation results of pinning control are shown in Figure 3.

In Figure 1, the error variables are divergent, which indicates that the original system is not synchronized. In Figures 2 and 3, the error variables converge to zero, which indicates that both the state feedback control and the pinning control can synchronize the network. However, the effects of state feedback control and pinning control have no obvious difference.

\section{Conclusion}

In this paper, the synchronization and control problem of linearly coupled singular systems has been investigated. 


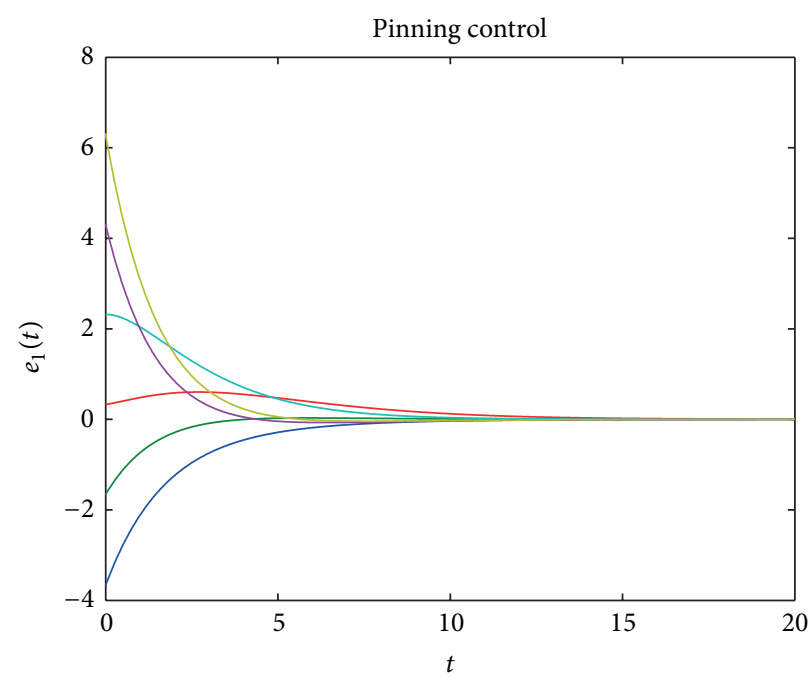

(a)

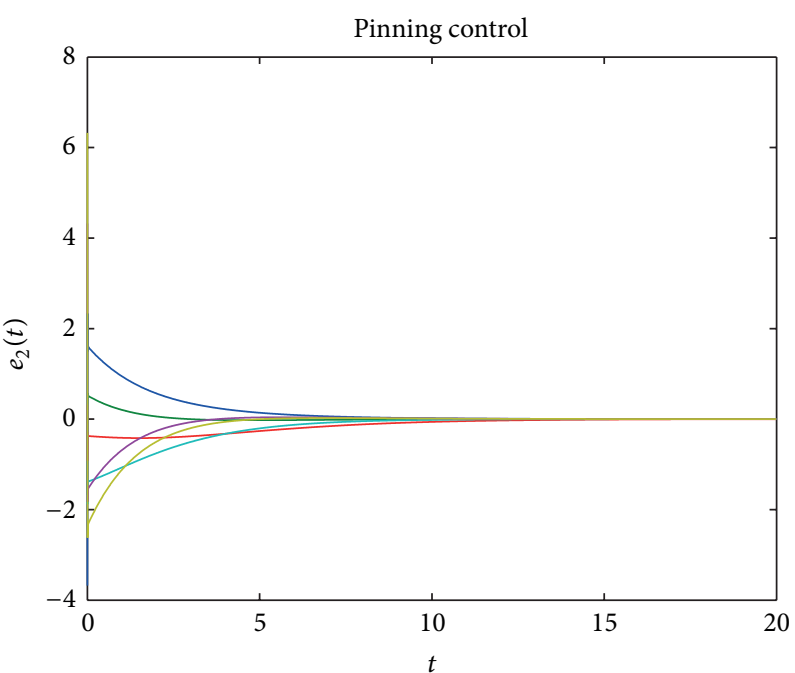

(b)

FIgURE 3: Curves of error variable $e_{i}(t)(i=1,2, \ldots, 6)$ of system (41).

The dynamical behaviors at each node and the coupling configuration are general and the coupling matrix is not assumed symmetrical. A reference state was proposed making use of the left eigenvector associated with eigenvalue zero of the coupling matrix, and some criteria for globally exponential synchronization were obtained based on the Lyapunov stability theory. The controllers are designed for state feedback control and pinning control to achieve globally exponential synchronization.

The significance of the paper, we think, can be summarized as follows. (1) The present paper applies a special reference state to linearly coupled singular systems and investigates the synchronization and control problem of these systems. Such work is rare in the existing literature to our knowledge. (2) The conditions adopted in this paper are described by strict linear matrix inequalities and can be solved directly by LMI toolbox of Matlab.

However, there are many problems unsolved about the synchronization and control problems of linearly coupled singular systems. For example, in this paper, only the linear coupling case was treated, and the nonlinear coupling case has not been involved; for the dynamical behaviors at each node, we only considered the regular singular systems, and the nonregular singular system case needs to be further studied.

\section{Acknowledgments}

This work was supported by NCET and partially by the NSFC under Grant 10531030 and 90818020 and Research Project of Zhejiang Province Education Department under Grant Y201018827.

\section{References}

[1] A. T. Winfree, The Geometry of Biological Time, vol. 8, Springer, New York, NY, USA, 1980.

[2] M. Dhamala, V. K. Jirsa, and M. Ding, "Transitions to synchrony in coupled bursting neurons," Physical Review Letters, vol. 92, no. 2, Article ID 028101, 4 pages, 2004.

[3] A. L. Lloyd and V. A. A. Jansen, "Spatiotemporal dynamics of epidemics: synchrony in metapopulation models," Mathematical Biosciences, vol. 188, no. 1-2, pp. 1-16, 2004.

[4] V. Perez-Munuzuri, V. Perez-Villar, and L. O. Chua, "Autowaves for image processing on a two-dimensional CNN array of excitable nonlinear circuits: flat and wrinkled labyrinths," IEEE Transactions on Circuits and Systems I, vol. 40, no. 3, pp. 174-181, 1993.

[5] L. M. Pecora and T. L. Carroll, "Synchronization in chaotic systems," Physical Review Letters, vol. 64, no. 8, pp. 821-824, 1990.

[6] R. E. Mirollo and S. H. Strogatz, "Synchronization of pulsecoupled biological oscillators," SIAM Journal on Applied Mathematics, vol. 50, no. 6, pp. 1645-1662, 1990.

[7] J. F. Heagy, T. L. Carroll, and L. M. Pecora, "Synchronous chaos in coupled oscillator systems," Physical Review E, vol. 50, no. 3, pp. 1874-1885, 1994.

[8] X. F. Wang and G. Chen, "Synchronization in scale-free dynamical networks: robustness and fragility," IEEE Transactions on Circuits and Systems I, vol. 49, no. 1, pp. 54-62, 2002.

[9] X. F. Wang and G. Chen, "Synchronization in small-world dynamical networks," International Journal of Bifurcation and Chaos in Applied Sciences and Engineering, vol. 12, no. 1, pp. 187192, 2002.

[10] C. Posadas-Castillo, C. Cruz-Hernandez, and R. M. LopezGutierrez, "Experimental realization of synchronization in complex networks with Chua's circuits like nodes," Chaos, Solitons and Fractals, vol. 40, pp. 1963-1975, 2009. 
[11] M. Lashmanan and K. Murali, Chaos in Nonlinear Oscillator: Controlling and Synchronization, World Scientific, Singgapore, 1996.

[12] C. Huygens, Horoloquium Oscillatorium, F. Muguet, Paris, France, 1672.

[13] Z. Néda, E. Ravasz, Y. Brechet, T. Vicsek, and A. L. Barabási, "The sound of many hands clapping," Nature, vol. 403, no. 6772, pp. 849-853, 2000.

[14] L. Kuhnert, K. I. Agladze, and V. I. Krinsky, "Image processing using light-sensitive chemical waves," Nature, vol. 337, no. 6204, pp. 244-247, 1989.

[15] V. I. Krinsky, V. N. Biktashev, and I. R. Efimov, "Autowave principles for parallel image processing," Physica D, vol. 49, no. 1-2, pp. 247-253, 1991.

[16] W. Lu and T. Chen, "Synchronization of coupled connected neural networks with delays," IEEE Transactions on Circuits and Systems I, vol. 51, no. 12, pp. 2491-2503, 2004.

[17] G. D. VanWiggeren and R. Roy, "Communication with chaotic lasers," Science, vol. 279, no. 5354, pp. 1198-1200, 1998.

[18] R. M. Lopez-Gutierrez, C. Posadas-Castillo, D. Lopez-Mancilla, and C. Cruz-Hernandez, "Communicating via robust synchronization of chaotic lasers," Chaos, Solitons and Fractals, vol. 42, pp. 277-285, 2009.

[19] C. Cruz-Hernandez, R. M. Lopez-Gutierrez, A. Y. AguilarBustos, and C. Posadas-Castillo, "Communicating encrypted information based on synchronized hyperchaotic maps," International Journal of Nonlinear Sciences and Numerical Simulation, vol. 11, pp. 337-349, 2010.

[20] F. C. Hoppensteadt and E. M. Izhikevich, "Pattern recognition via synchronization in phase-locked loop neural networks," IEEE Transactions on Neural Networks, vol. 11, no. 3, pp. 734$738,2000$.

[21] C. W. Wu and L. O. Chua, "Synchronization in an array of linearly coupled dynamical systems," IEEE Transactions on Circuits and Systems I, vol. 42, no. 8, pp. 430-447, 1995.

[22] H. Nijmeijer and I. M. Y. Mareels, "An observer looks at synchronization," IEEE Transactions on Circuits and Systems I, vol. 44, no. 10, pp. 882-890, 1997.

[23] L. Pecora, T. Carroll, G. Johnson, M. Doug, and K. S. Fink, "Synchronization stability in coupled oscillator arrays: solution for arbitrary configurations," International Journal of Bifurcation and Chaos in Applied Sciences and Engineering, vol. 10, no. 2, pp. 273-290, 2000.

[24] W. Lu and T. Chen, "New approach to synchronization analysis of linearly coupled ordinary differential systems," Physica D, vol. 213, no. 2, pp. 214-230, 2006.

[25] C. Liu, Z. Duan, G. Chen, and L. Huang, " $L_{2}$ norm performance index of synchronization and LQR control synthesis of complex networks," Automatica, vol. 45, no. 8, pp. 1879-1885, 2009.

[26] J. Lu and D. W. C. Ho, "Globally exponential synchronization and synchronizability for general dynamical networks," IEEE Transactions on Systems, Man, and Cybernetics B, vol. 40, no. 2, pp. 350-361, 2010.

[27] H. Liu, J. Chen, J. A. Lu, and M. Cao, "Generalized synchronization in complex dynamical networks via adaptive couplings," Physica A, vol. 389, no. 8, pp. 1759-1770, 2010.

[28] C. X. Fan, G. P. Jiang, and F. H. Jiang, "Synchronization between two complex dynamical networks using scalar signals under pinning control," IEEE Transactions on Circuits and Systems I, vol. 57, no. 11, pp. 2991-2998, 2010.
[29] Z. H. Guan, Z. W. Liu, G. Feng, and Y. W. Wang, "Synchronization of complex dynamical networks with time-varying delays via impulsive distributed control," IEEE Transactions on Circuits and Systems I, vol. 57, no. 8, pp. 2182-2195, 2010.

[30] W. Xiong, D. W. C. Ho, and J. Cao, "Synchronization analysis of singular hybrid coupled networks," Physics Letters A, vol. 372, no. 44, pp. 6633-6637, 2008.

[31] L. Y. Dai, “Observers for discrete singular systems," Transactions on Automatic Control, vol. 33, no. 2, pp. 187-191, 1988.

[32] J. D. Aplevich, Implicit Linear Systems, Springer, Berlin, 1991.

[33] E. Uezato and M. Ikeda, "Strict LMI conditions for stability, robust stabilization, and $H_{\infty}$ control of descriptor systems," in Proceedings of the 38th IEEE Conference on Decision and Control (CDC '99), pp. 4092-4097, December 1999.

[34] S. Xu and C. Yang, " $H_{\infty}$ state feedback control for discrete singular systems," IEEE Transactions on Automatic Control, vol. 45, no. 7, pp. 1405-1409, 2000.

[35] X. Liu and D. W. C. Ho, "Disturbance decoupling of linear timevarying singular systems," IEEE Transactions on Automatic Control, vol. 47, no. 2, pp. 335-341, 2002.

[36] S. Xu, P. van Dooren, R. Ştefan, and J. Lam, "Robust stability and stabilization for singular systems with state delay and parameter uncertainty," IEEE Transactions on Automatic Control, vol. 47, no. 7, pp. 1122-1128, 2002.

[37] L. Zhang, B. Huang, and J. Lam, "LMI synthesis of $\mathrm{H}_{2}$ and mixed $\mathrm{H}_{2} / \mathrm{H}_{\infty}$ controllers for singular systems," IEEE Transactions on Circuits and Systems II, vol. 50, no. 9, pp. 615-626, 2003.

[38] G. Lu and D. W. C. Ho, "Continuous stabilization controllers for singular bilinear systems: the state feedback case," Automatica, vol. 42, no. 2, pp. 309-314, 2006.

[39] B. Meng and J. F. Zhang, "Reachability conditions for switched linear singular systems," IEEE Transactions on Automatic Control, vol. 51, no. 3, pp. 482-488, 2006.

[40] G. Lu and D. W. C. Ho, "Generalized quadratic stability for continuous-time singular systems with nonlinear perturbation," IEEE Transactions on Automatic Control, vol. 51, no. 5, pp. 818823, 2006.

[41] G. Zhang, Y. Xia, and P. Shi, "New bounded real lemma for discrete-time singular systems," Automatica, vol. 44, no. 3, pp. 886-890, 2008.

[42] G. Grammel, "Robustness of exponential stability to singular perturbations and delays," Systems and Control Letters, vol. 57, no. 6, pp. 505-510, 2008.

[43] L. Lv and Z. Lin, "Analysis and design of singular linear systems under actuator saturation and $L_{2} / L_{\infty}$ disturbances," Systems and Control Letters, vol. 57, no. 11, pp. 904-912, 2008.

[44] A. Haidar and E. K. Boukas, "Exponential stability of singular systems with multiple time-varying delays," Automatica, vol. 45, no. 2, pp. 539-545, 2009.

[45] I. Masubuchi, Y. Kamitane, A. Ohara, and N. Suda, " $H_{\infty}$ control for descriptor systems: a matrix inequalities approach," Automatica, vol. 33, no. 4, pp. 669-673, 1997.

[46] F. L. Lewis, "A survey of linear singular systems," Circuits, Systems, and Signal Processing, vol. 5, no. 1, pp. 3-36, 1986. 


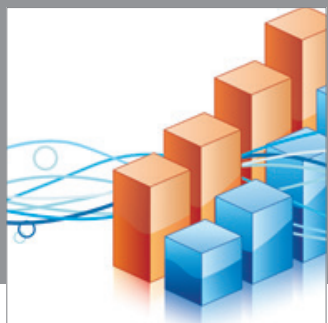

Advances in

Operations Research

mansans

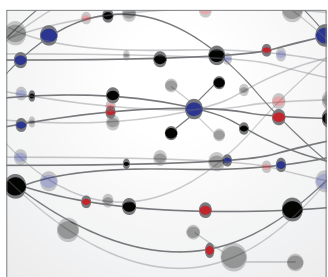

The Scientific World Journal
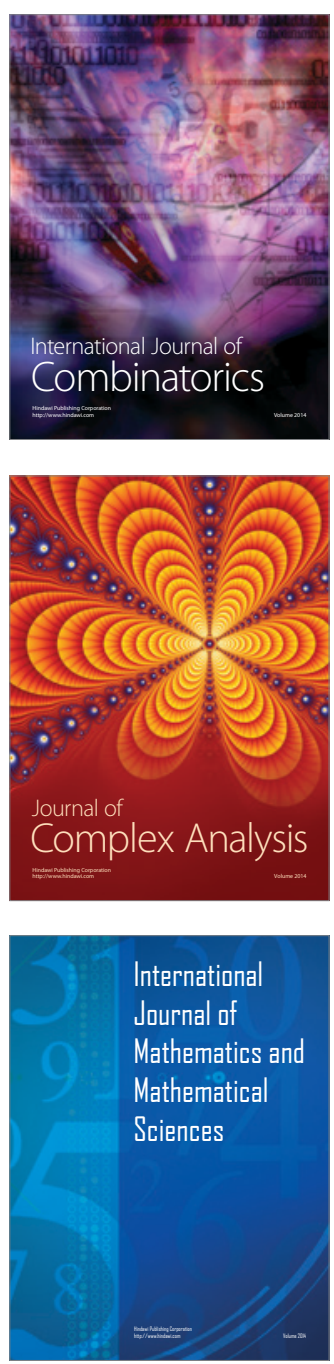
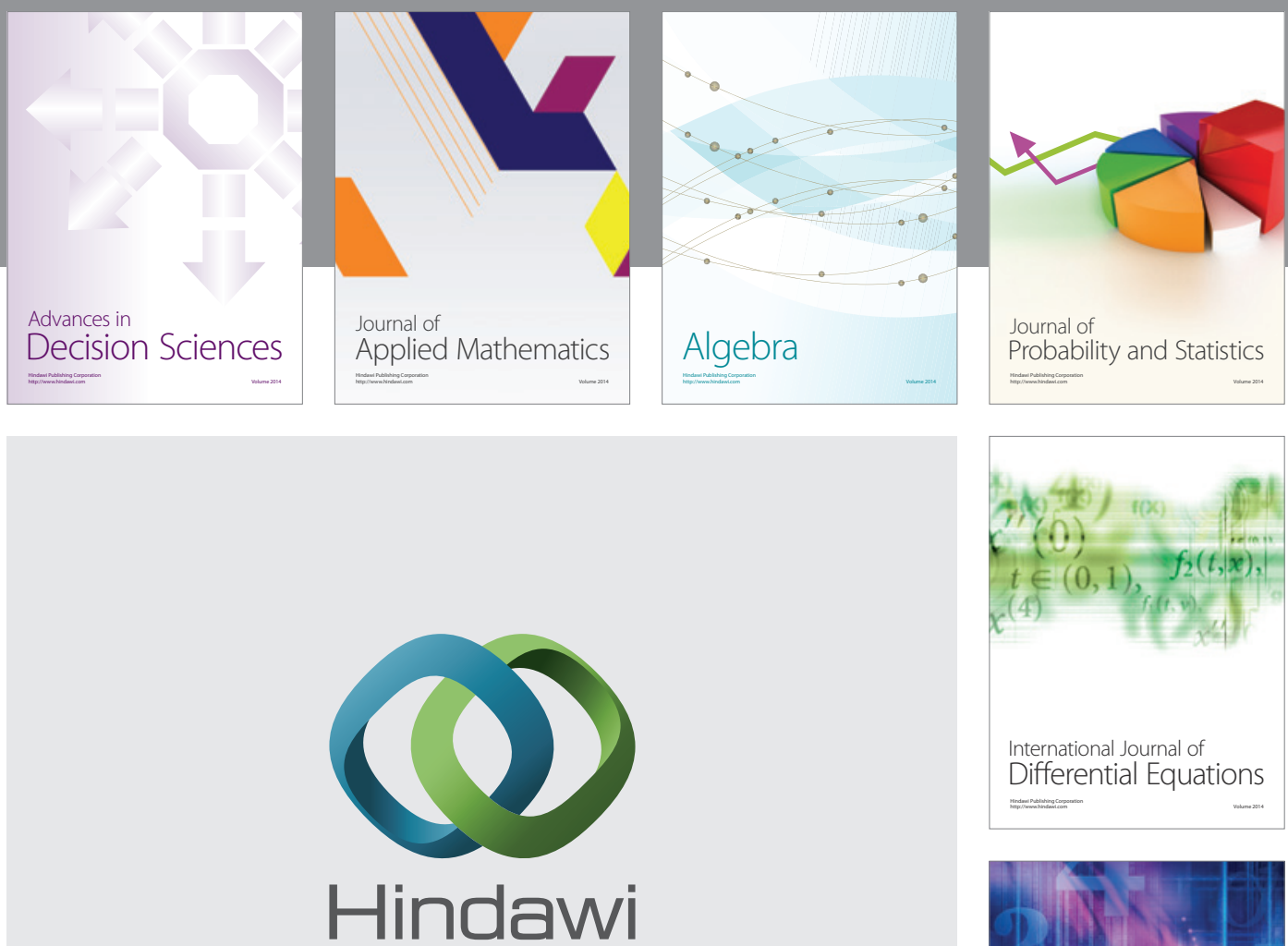

Submit your manuscripts at http://www.hindawi.com
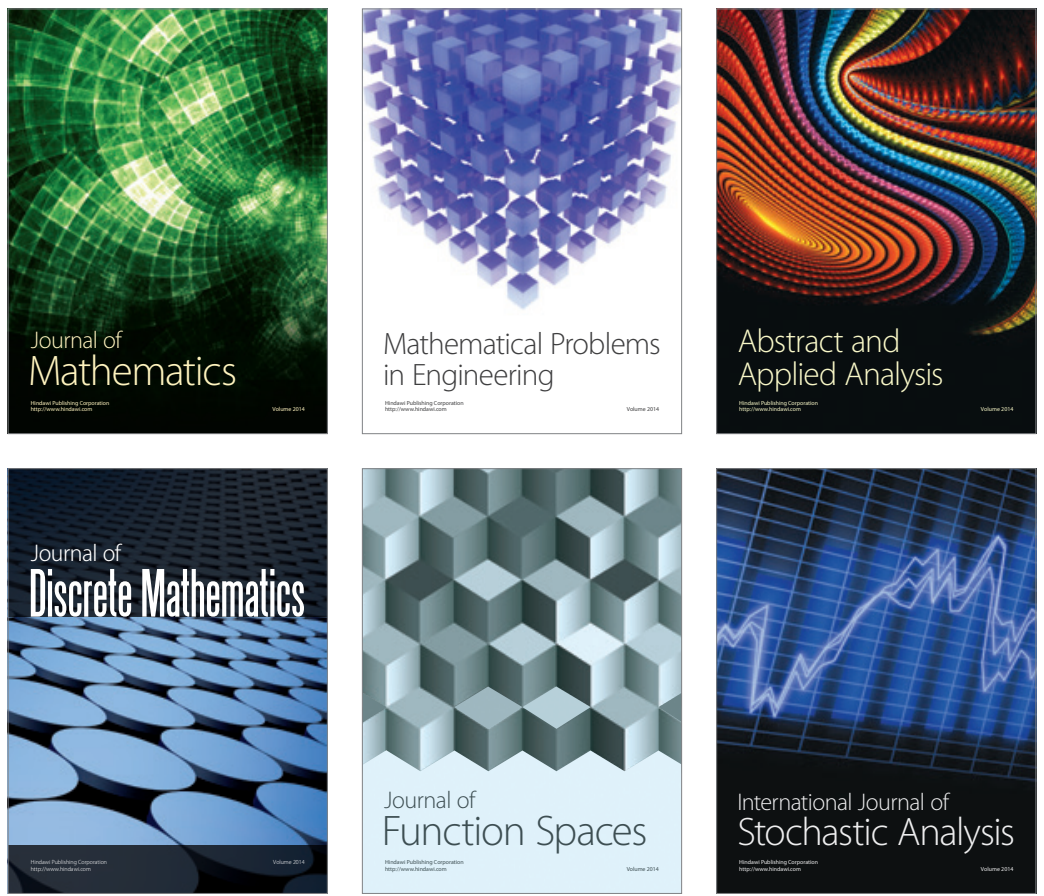

Journal of

Function Spaces

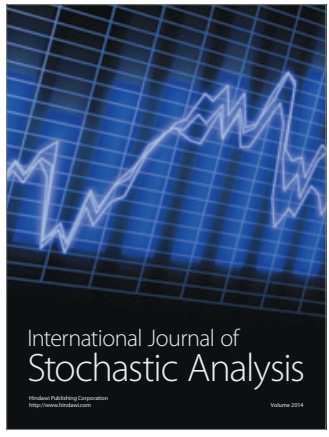

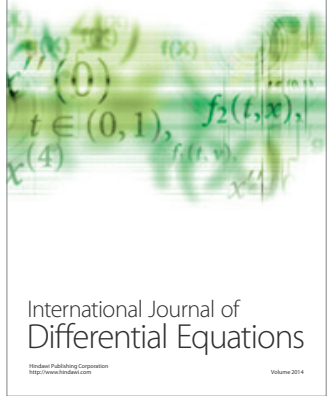
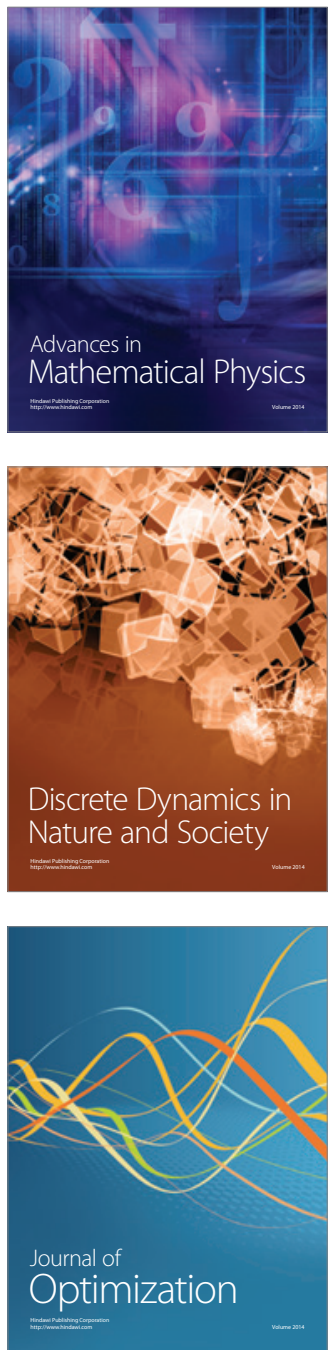